\title{
IMAGE DEPTH PROFILING AES
}

\author{
T. SEKine ANd T. Sato \\ Jeol Ltd., 3-1-2 Musashino, Akishima, Tokyo 196, Japan \\ (Received May 21, 1991; in revised form August 10, 1991)
}

\begin{abstract}
Image depth profiling AES incorporating an image processing technique has been developed. This method reconstructs a cross-sectional image from acquired 2-dimensional and/or 3-dimensional data. A binary image produced from a secondary electron image through image processing can be used to cut the area of interest for selected area analysis. In a failure analysis of a relay contact, we found by cross-sectional image observation that $\mathrm{Fe}$ and $\mathrm{Ni}$ permeate from pin holes in an Au layer covering a Fe-Ni alloy. A VTR head made of a polycrystalline ferrite core covered with a Sendust (Si-Al-Fe alloy) layer at the gap has been analyzed. Depth distributions of $\mathrm{Al}, \mathrm{O}$, and $\mathrm{Fe}$ on different grains, and $\mathrm{Al}$ and $\mathrm{O}$ distributions near grain boundaries have been successfully reassembled from the 3-dimensional data.
\end{abstract}

PACS numbers: 68.35.Dv

\section{Introduction}

Chemical control at surfaces and interfaces is a key issue in modern materials science and technology. This control requires reliable chemical analysis with respect to distance from the surface or interface. Sputter depth profiling by Auger electron spectroscopy (AES) is one of the most effective means for determining the depth distribution of chemical composition in surface layers. Usually, it is applied to one or several points on the specimen surface. However, the information on a limited number of representative points is not enough for observing all the characteristics of the specimen when it has lateral inhomogeneities or small structures. In such cases, we sometimes find an unexpected scatter of measured data at the beginning of analysis. And we often cannot interpret the reason of this scattering until we conduct a number of measurements. In secondary ion mass spectroscopy (SIMS), reconstruction of a cross-sectional image from a number of accumulated secondary ion images during sputtering has been attempted for many years [1, 2]. The same idea can be utilized for solving the above-mentioned problem by AES as well. We have developed image depth profiling AES incorporating image processing techniques. Details and applications are shown in the following paragraphs. 


\section{Methods of image depth profiling}

A conceptional diagram for image depth profiling is shown in Fig. 1. Two methods are illustrated together with the conventional depth profiling method. The conventional method shown in Fig. 1a gives us average compositional information on the spot area where the electron beam is irradiated. On the other
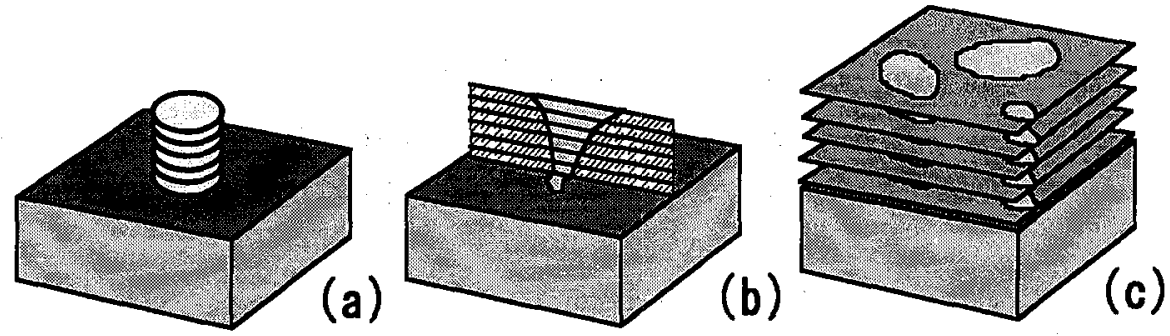

Fig. 1. Three methods of depth profiling. (a) Conventional method of depth profile acquisition. Average information from a spot area is obtained. (b) 2-D depth profiling. A cross-sectional image is obtained. (c) 3-D depth profiling. A cross-sectional image through a given line, an image at a given depth, and a local area depth profile are constructed through post-acquisition processing.

hand, the image depth profiling method gives us both lateral and depth distributions at once. One method of image depth profiling is illustrated in Fig. 1b. This method repeats measurement of a line profile along a given line and ion sputtering on the specimen surface alternately, and stores the collected data into files. The line profiles show the intensity distribution at each depth level along the line. Then, by post-acquisition processing, a cross-sectional image can be generated from the stored data [3]. Both Auger electron images (Auger images) and secondary electron images (SEIs) can be handled. This is actually a two-dimensional (2-D) analysis. In the second method shown in Fig. 1c, imaging instead of line profiling and ion sputtering are carried out alternately. The stored images provide three-dimensional (3-D) information. There are many ways to view the acquired 3-D data. For example, a cross-sectional image can be constructed by looking at the intensity distribution along a line for each of the stored images. Another procedure to process image depth profile data is to produce a depth profile using only the signal from a specified region of the image.

We have developed a simple analytical language for automated operation. 2-D and 3-D data acquistions are carried out by this language automatically.

\section{Applications}

\subsection{Failure analysis of relay contact}

The relay switch is a well-used part in electric circuits. One of its typical structures is illustrated in Fig. 2a. In this example, a pair of electrodes made of $\mathrm{Fe}-\mathrm{Ni}$ alloy, with each surface plated with an Au layer of $700 \mathrm{~nm}$ thickness, is 


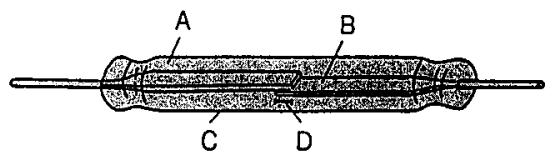

(a)

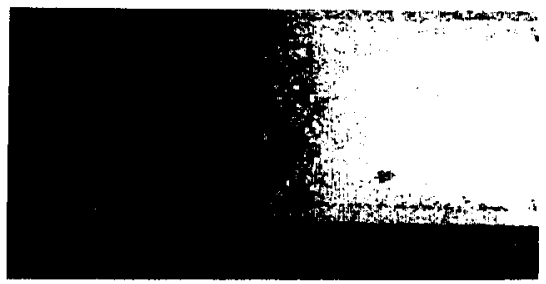

(c)

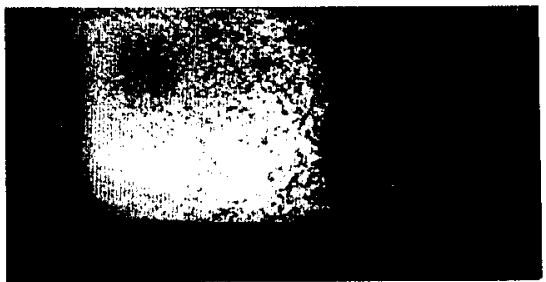

(e)

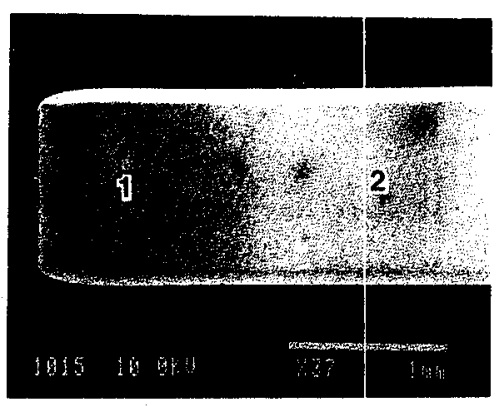

(b)

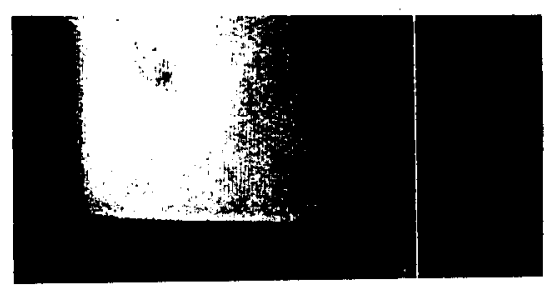

(d)

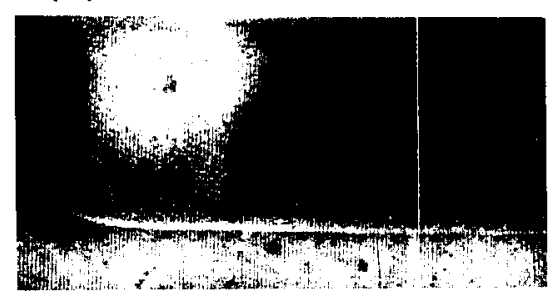

(f)

Fig. 2. (a) Structure of relay switch A: sealed with nitrogen gas; B: electrode piece; C: glass tube; D: contact point. (b) Secondary electron image on electrode piece surface of relay switch. 1: contact area; 2: non-contact area. (c) Au-NOO Auger electron image. (d) Fe- $L M M$ Auger electron image. (e) Ni- $L M M$ Auger electron image. (f) O- $K L L$ Auger electron image.

sealed in a glass tube filled with $\mathrm{N}_{2}$ gas. The switch is magnetically opened or closed from outside the tube. The relay may develop improper contact after a number of repeated operations over a long time. Diffusion of $\mathrm{Fe}$ and $\mathrm{Ni}$ into an $\mathrm{Au}$ layer is known as a cause of contact failure [4-6]. These elements segregate to the surface and can be oxidized to give a layer of high resistance. A SEI of an electrode is shown in Fig. 2b. The contact area (labeled with "1") looks slightly darker than the non-contact area (labeled with " 2 "). This surface was first investigated by Auger image analysis. The $\mathrm{Au}$ which should cover the surface was not detected near the area " 1 ", while $\mathrm{Fe}, \mathrm{Ni}$ and $\mathrm{O}$ were found there (Fig. 2c-f). At the non-contact area 
"2", Au was clearly detected (Fig. 2c). The depth profile shown in Fig. 3 proves that the existence of $\mathrm{Fe}$ and $\mathrm{Ni}$ is not because of the loss of $\mathrm{Au}$, but because of the diffusion of $\mathrm{Fe}$ and $\mathrm{Ni}$ to the surface. We then studied whether $\mathrm{Fe}$ and $\mathrm{Ni}$

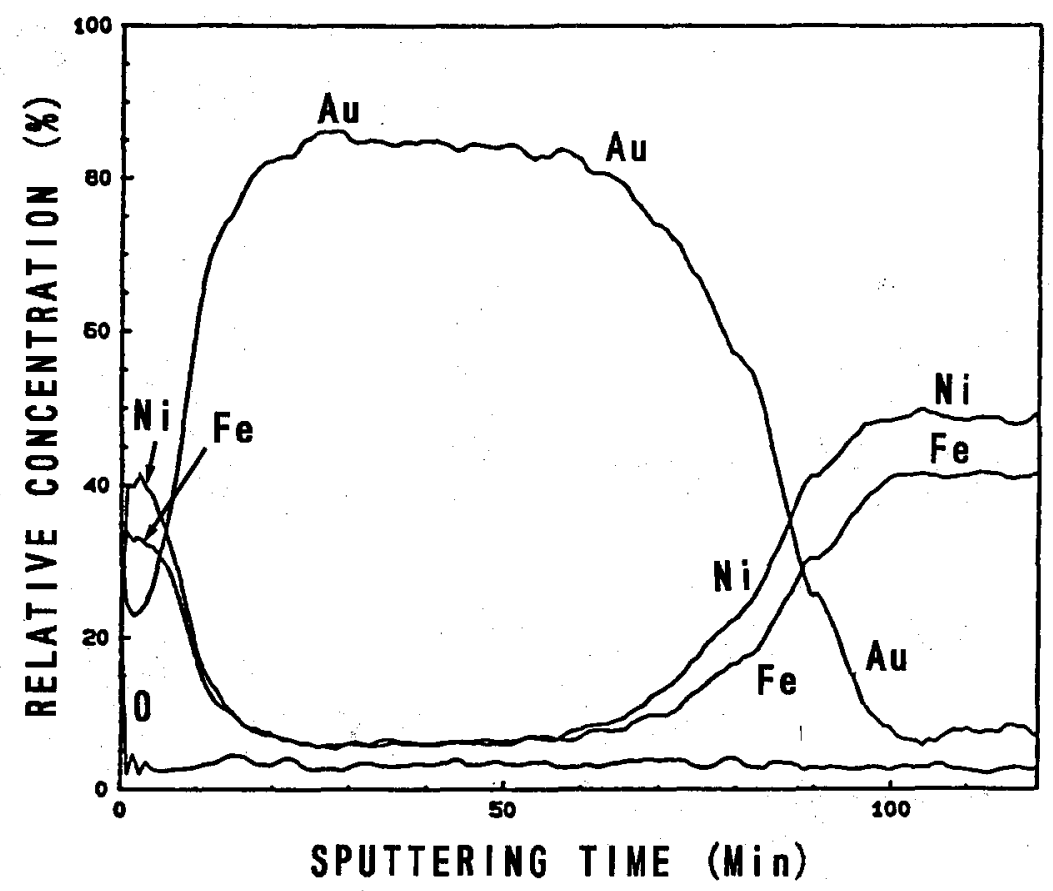

Fig. 3. Depth profile at contact area by conventional method.

diffused through the Au layer uniformly or by specific routes. For this purpose, 2-D analysis was applied. Cross-sectional images of $\mathrm{Au}, \mathrm{Fe}$, and $\mathrm{Ni}$ were constructed from the collected data on the line shown in the image (Fig. 4a). They are shown in Fig. 4c-e, respectively. The cross-sectional images show clearly that $\mathrm{Fe}$ and $\mathrm{Ni}$ diffused through specific pin holes (perhaps gaps between column-like structures). The area containing diffused $\mathrm{Fe}$ and $\mathrm{Ni}$ look brighter than the rest of the areas in an SEI obtained after the depth profiling measurement (120 min sputtering) as shown in Fig. 4b.

Comparing the $\mathrm{Fe}$ and $\mathrm{Ni}$ images, we can see that $\mathrm{Fe}$ has diffused more than $\mathrm{Ni}$. A ternary composite image of $\mathrm{Fe}, \mathrm{Ni}$ and $\mathrm{Au}$ helps to find variations of relative intensities among the elements (Fig. 4f). We find from the color change at the interface region that an $\mathrm{Ni}$ rich zone is formed there. This zone is due to more $\mathrm{Fe}$ than $\mathrm{Ni}$ diffusing into the $\mathrm{Au}$ layer (the darker blue at the interface indicates that $\mathrm{Ni}$ is rich there). 


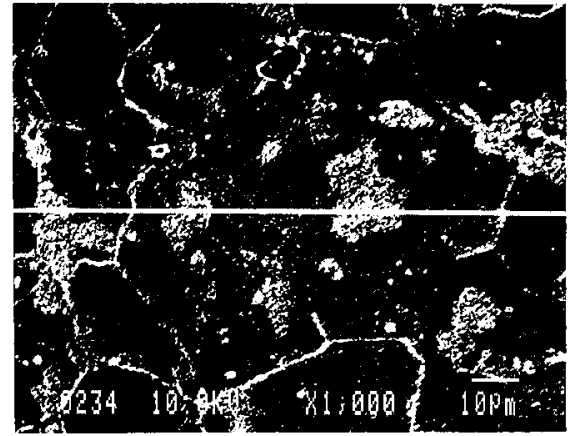

(a)

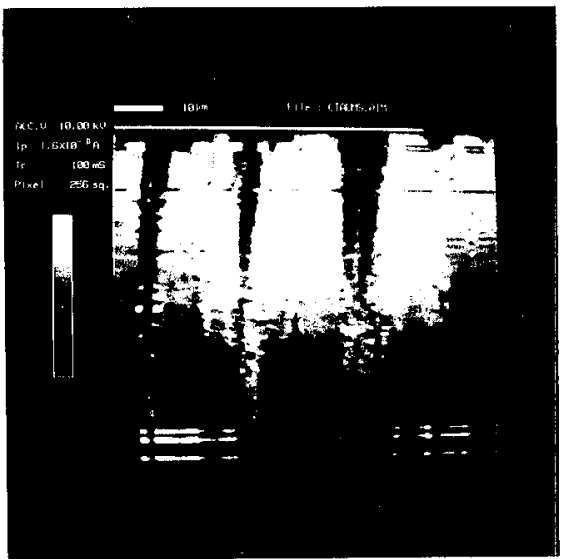

(c)

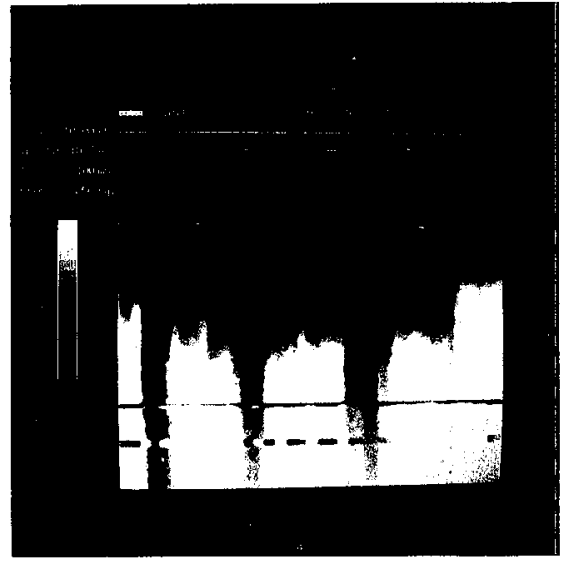

(e)

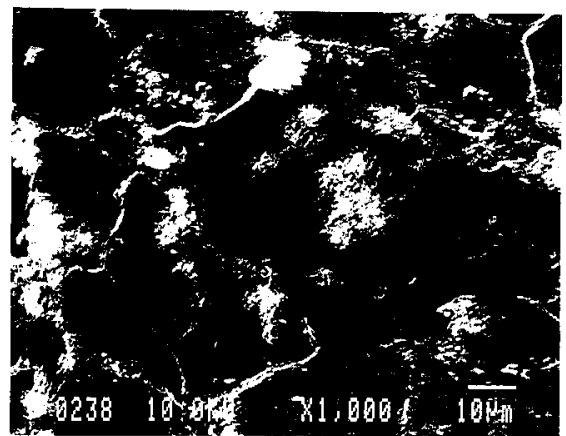

(b)

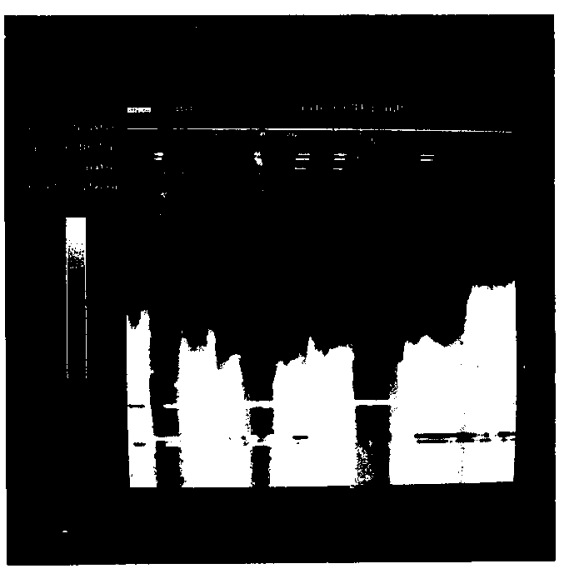

(d)

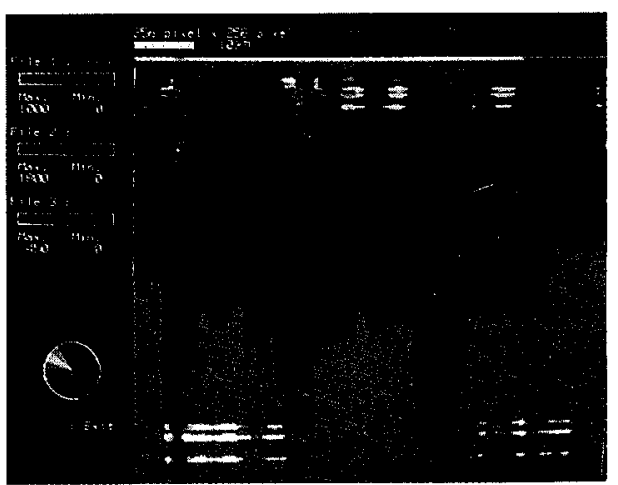

Fig. 4. (a) Secondary electron image at contact area (before ion sputtering). Depth profile images on the cross-section through the line shown in the image were measured. (b) Secondary electron image at contact area (after ion sputtering of $700 \mathrm{~nm}$ ). (d) Fe- $M N N$ cross-sectional image. (e) Ni- $M N N$ cross-sectional image. (f) Ternary composite image of cross-section (original photograph was shown in color, green: $\mathrm{Fe}$, blue: $\mathrm{Ni}$, red: $\mathrm{Au})$. 


\subsection{Analysis of VTR magnetic head}

For the video tape recorder (VTR), intensive development has been continued in order to realize higher recording density [7]. Various types of recording heads have been developed and are actually used. There is a magnetic head consisting of a ferrite core with a gap on which an alloy called "Sendust" is deposited. Sendust is a Fe-based alloy that has a high permeability for magnetic flux. In this type of head, a pseudogap between the Sendust and the ferrite is sometimes formed. This gap causes problems through the generation of pseudosignals. Therefore, materials that does not form a pseudogap have been developed. As a ferrite core, crystalline materials are preferable. However, polycrystalline ferrite is also used to reduce the production cost. In Fig. 5a, a head structure is illustrated. We have analyzed a portion of the gap by the 3-D method. A cross-section of the specimen studied is given in Fig. 5b. A Sendust layer (9.6wt.\%Si-5.5wt.\%Al-Fe) of $120 \mathrm{~nm}$ thickness is deposited on a polished manganese-zinc ferrite core $\left(\mathrm{Mn}_{x} \mathrm{Zn}_{1-x} \mathrm{Fe}_{2} \mathrm{O}_{4}\right)$ by sputtering. The ferrite consists of polycrystals with grains of about 10 micron

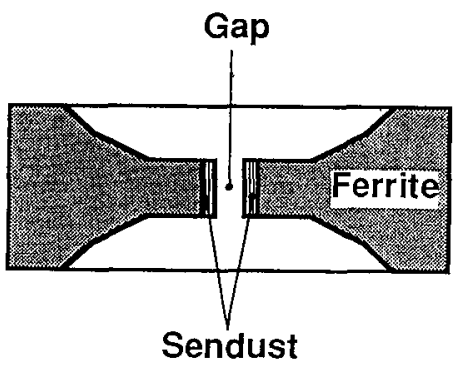

(a)

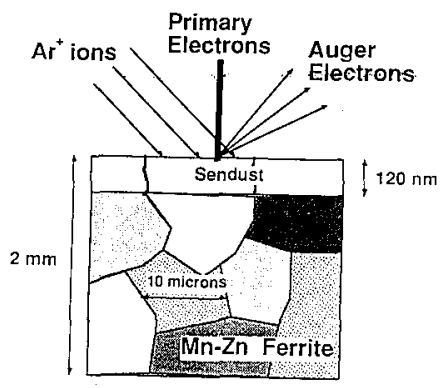

(b)

Fig. 5. (a) Structure of VTR recording head. (b) Cross-sectional drawing of analyzed specimen. Ferrite $\left(\mathrm{Mn}_{x} \mathrm{Zn}_{1-x} \mathrm{Fe}_{2} \mathrm{O}_{4}\right)$ is covered with Sendust layer (9.6wt\%Si-5.5wt.\%Al-Fe) of thickness $120 \mathrm{~nm}$ at the gap.

diameter (Fig. 5b). The specimen was annealed in a $\mathrm{N}_{2}$ atmosphere for 2 hours at a temperature of $600^{\circ} \mathrm{C}$ after the deposition. A depth profile obtained by the conventional method is shown in Fig. 6. In addition, 3-D data acquisition was carried out. Auger images of $\mathrm{Fe}, \mathrm{Al}$, and $\mathrm{O}$, and also SEIs at 30 depth levels were obtained by sputtering the specimen to a depth of $205 \mathrm{~nm}$. During sputtering, the specimen was rotated to remove surface atoms as uniformly as possible [8]. Images at every second depth level from the top surface to the 30th depth level are shown in Fig. 7. It is noted that the signal intensity varies from grain to grain in the Auger images and also in the SEIs. These changes are caused by the electron channeling effect in grains of different crystal orientation. It is known that rotating the specimen during sputtering greatly reduces the sputtering rate variations from place to place. However, we still see a small difference in the sputtering rate among the grains. Also, we note that the depth compositional distribution varies between the grains.

In order to study these variations more precisely, we have reproduced depth 


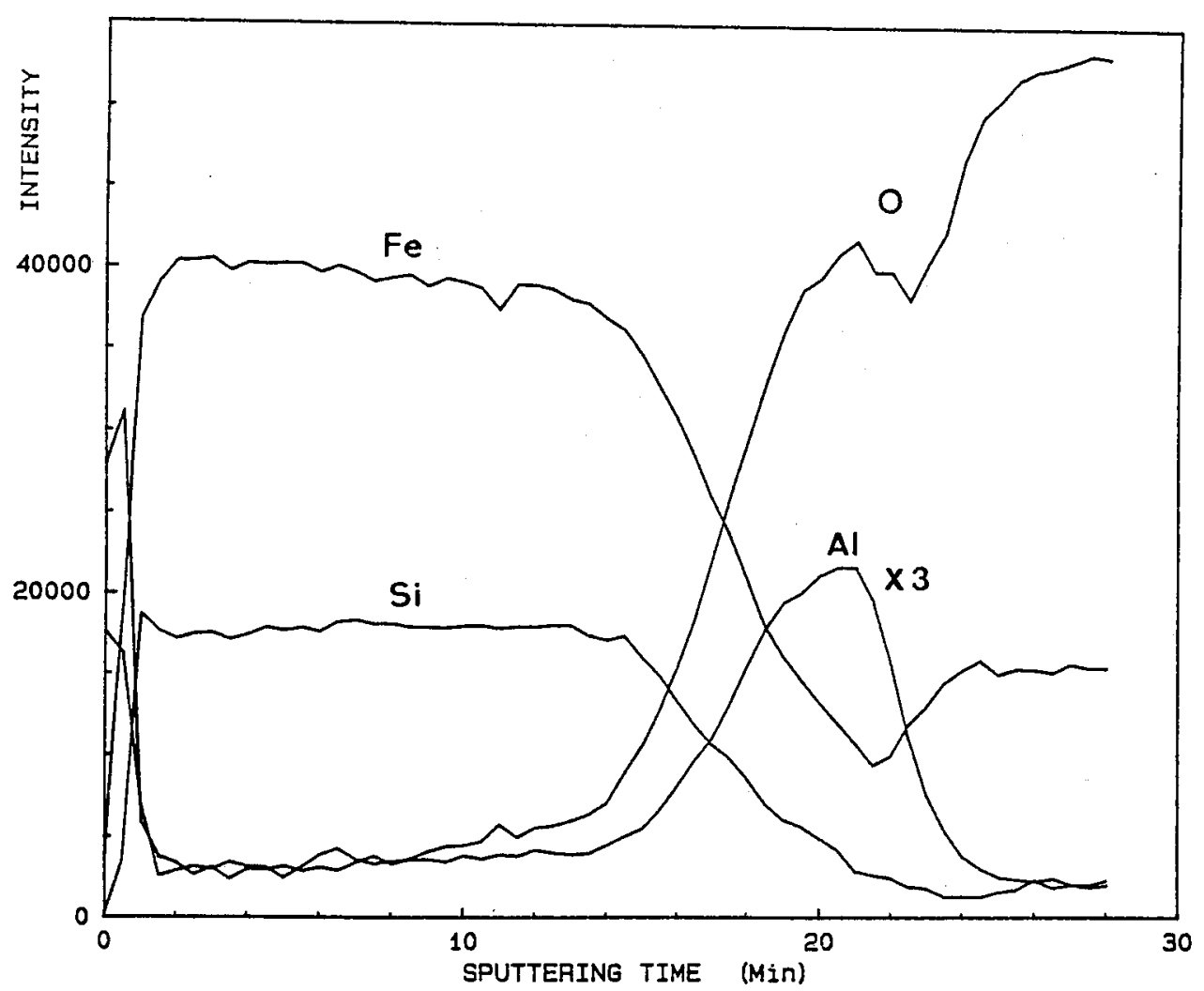

Fig. 6. Average in-depth composition of the Sendust obtained by the conventional method. The specimen was rotated during ion sputtering.

profiles of selected areas from the 3-D data. Three areas (G1, G2, and G3 shown in Fig. 8a) were analyzed. The areas G1 and G2 were selected by image processing from one of the SEIs. The reproduced depth profiles are indicated in Fig. 8b-d. On G1, as seen in Fig. 8b, an aluminum oxide layer is formed at the interface. On G2, the aluminum oxide layer is broader and split. At the center of the interface, Fe signal increases a little when the aluminum and oxygen signals decrease (Fig. 8c). G3 shows an intermediate state of these (Fig. 8d). The cross-sectional images generated from the 3-D data are shown in Fig. 9. Figures $9 \mathrm{~b}-\mathrm{d}$ are images corresponding to the lines 1, 2, and 3 shown in Fig. 9a, respectively. The Al layer (oxidized) formed at the interface is observed clearly. The line 2 is set to run through a large grain, small grains and a grain boundary. Al was detected along the grain boundary (Fig. 9c), while not so much $\mathrm{Al}$ was detected inside the large grain. The line 3 runs through small grains. It is seen that more $\mathrm{Al}$ is distributed at the area consisting of small grains (Fig. 9d). Also, the interface depth is shifted downward in the central portion of the image in Fig. 9d. This shift is due to a slower sputtering rate. It should be noted that a sputtered cross-section image will be distorted due to differences in sputtering rate in the different grains. 


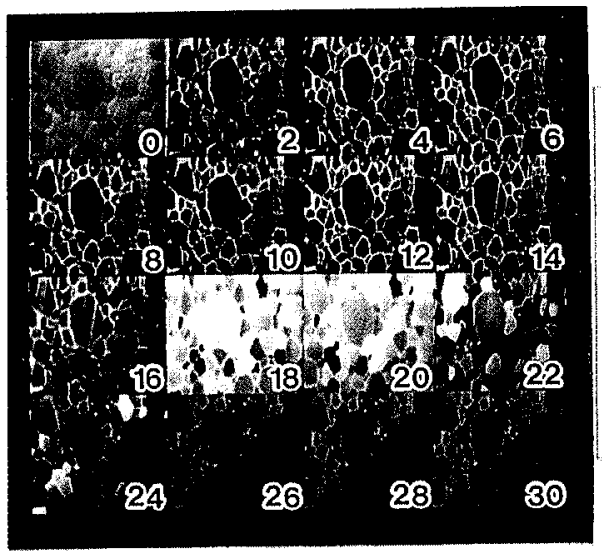

(a)

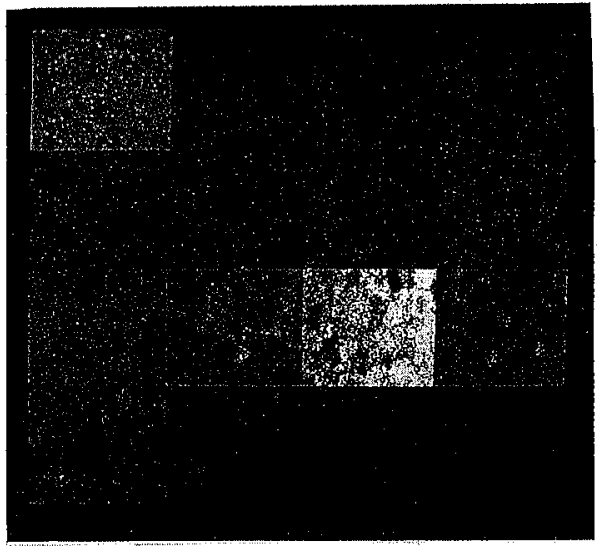

(c)

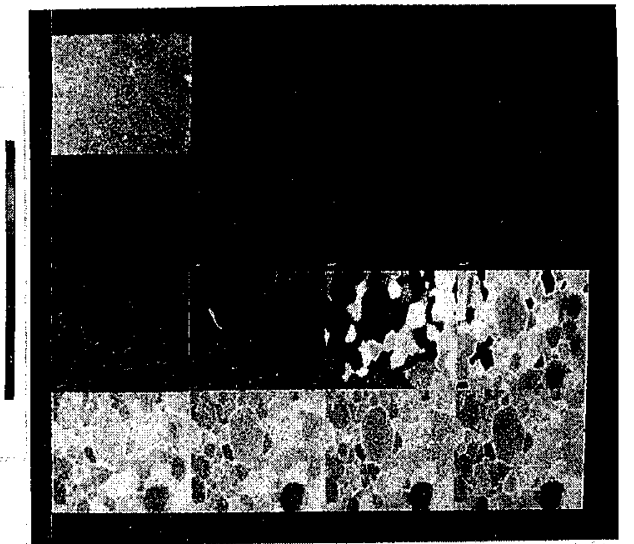

(b)

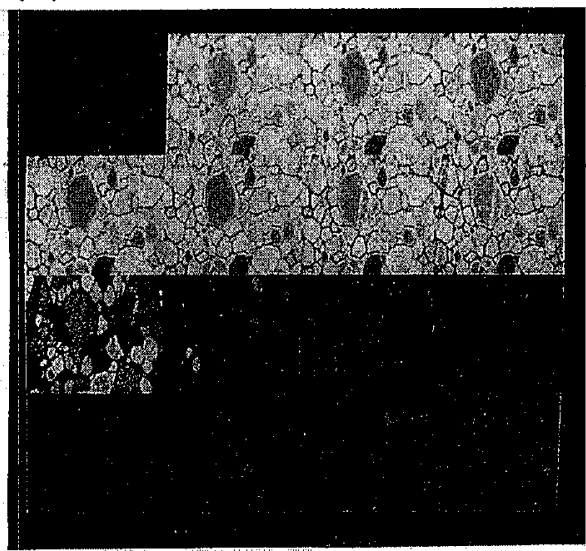

(d)

Fig. 7. Images of the Sendust at different depth levels obtained by 3-D sputter depth profile acquisition. Images were acquired after every $1 \mathrm{~min}$ of ion sputtering. (a) Secondary electron images. Figures in the images represent the accumulated sputtering time. The image marked with "0" is for the top surface. (b) O-Auger images. (c) Al-Auger images. (d) Fe-Auger images. Note (b)-(d): original photographs were shown in color.

We have studied the areas near grain boundaries more precisely. In order to accumulate signals from all grain boundaries in the 3-D data, we have generated an image mask (a binary image with unit intensity at the interface and zero at other places) by processing a secondary electron image. Firstly, we have carried out Laplacian filtration to get a differentiated image. This filter responds to changes in intensity so a new image is produced which shows high intensity at the grain boundaries. This image is transformed into a binary image after setting a certain threshold level. Noises and unwanted regions can be removed to some extent by this process. Secondly, we also obtained a binary image by slicing the intensity of 


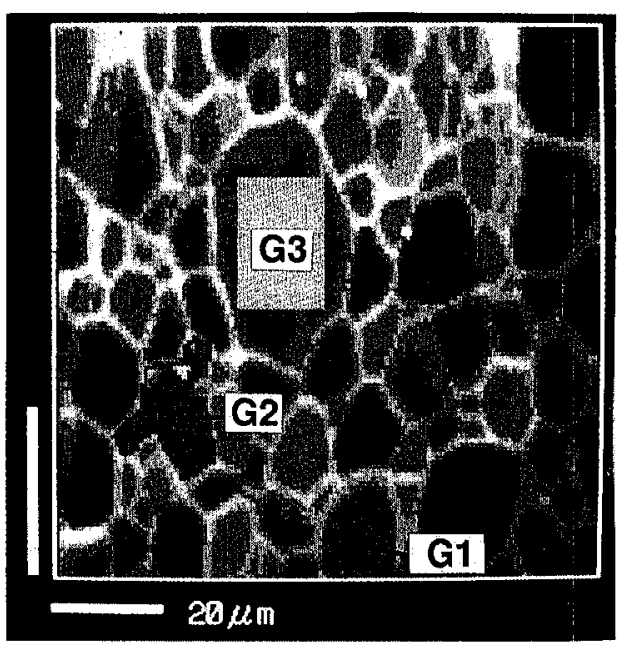

(a)

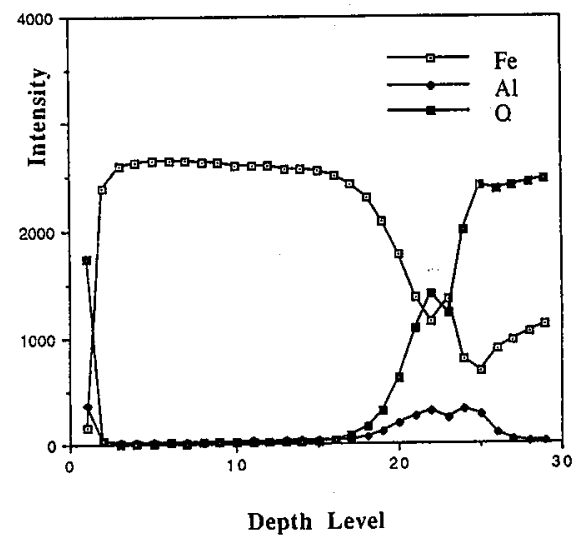

(c)

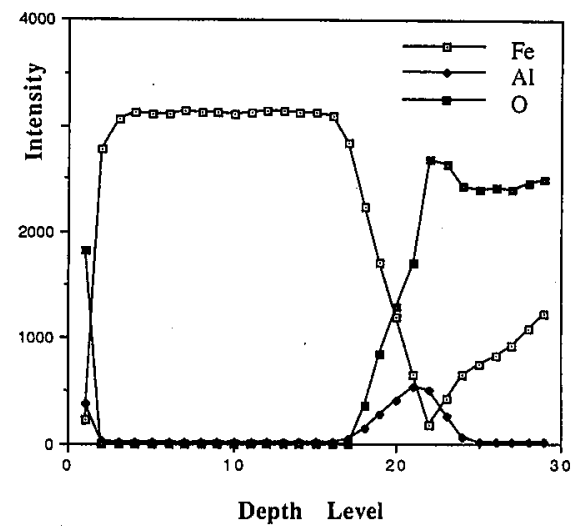

(b)

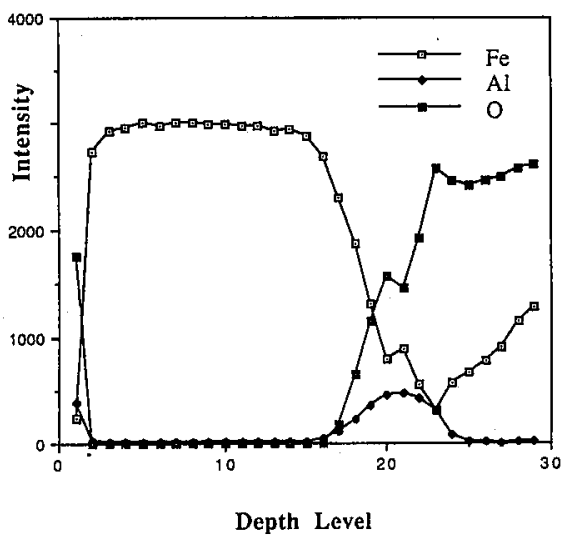

(d)

Fig. 8. Local area depth profiles reproduced from 3-D data for the Sendust. (a) Secondary electron image showing the selected areas (G1, G2, G3) where local depth profiles were produced. (b) Depth profile on G1. (c) Depth profile on G2. (d) Depth profile on G3.

the original SEI with a selected threshold level. This sliced binary image roughly corresponds to the grain boundary, however, it sometimes includes an unwanted region other than the grain boundary. Thirdly, to cut out a wanted region, we create a new image from the first and second binary images by taking a logical "AND". An example of processed image overlapped on the original SEI is shown 

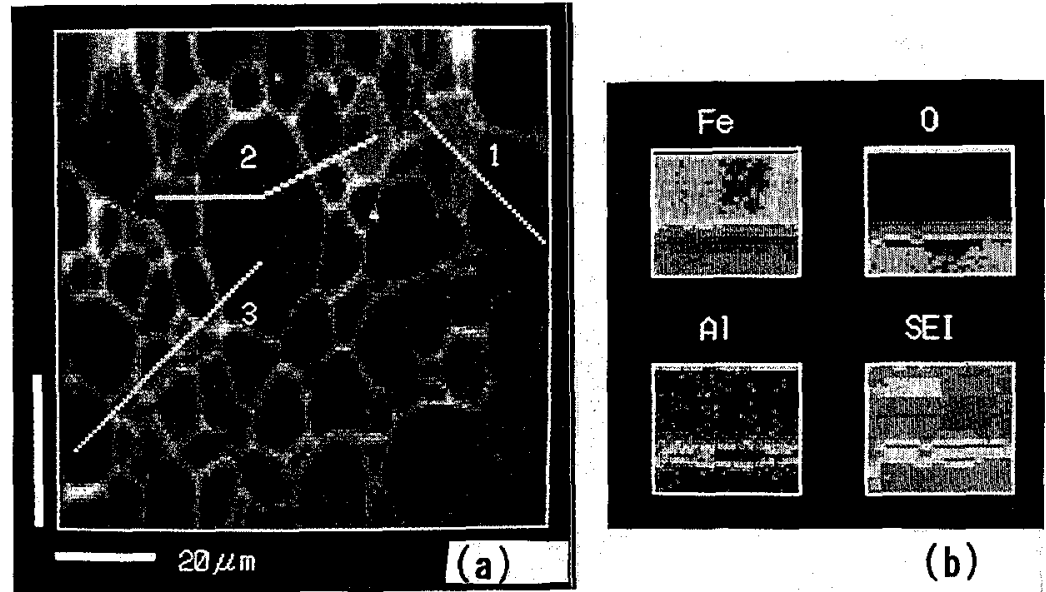

(b)
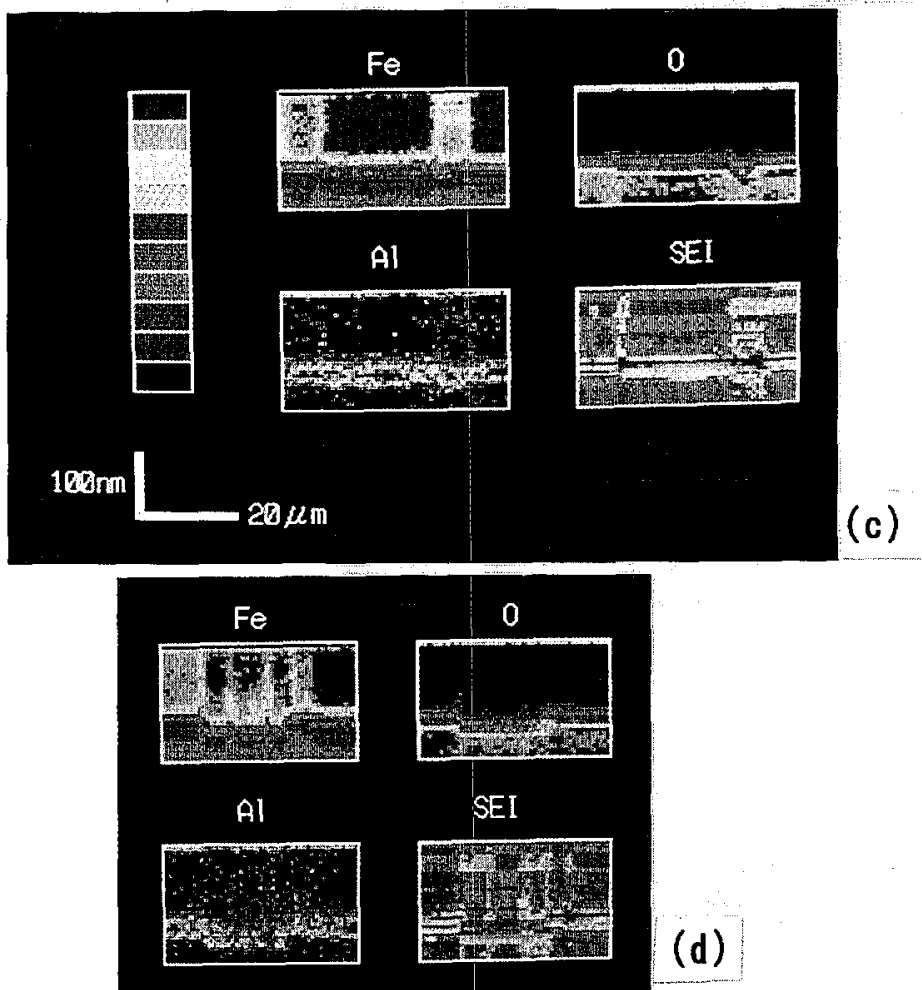

Fig. 9. Cross-sectional images of $\mathrm{Fe}, \mathrm{O}, \mathrm{Al}$ and the SEI for the Sendust. (a) The SEI showing the lines $(1,2,3)$ along which the cross-sectional images were generated. (b) Cross-sectional images along line 1. (c) Cross-sectional images along line 2. (d) Cross-sectional images along line 3 . Note (b)-(d): original photographs were shown in color. 


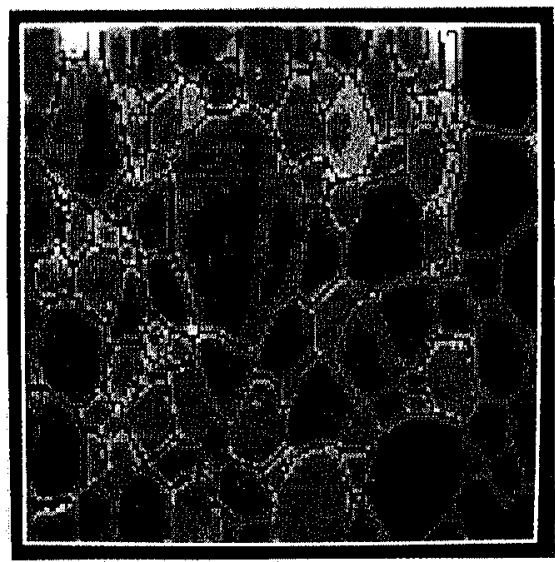

(a)
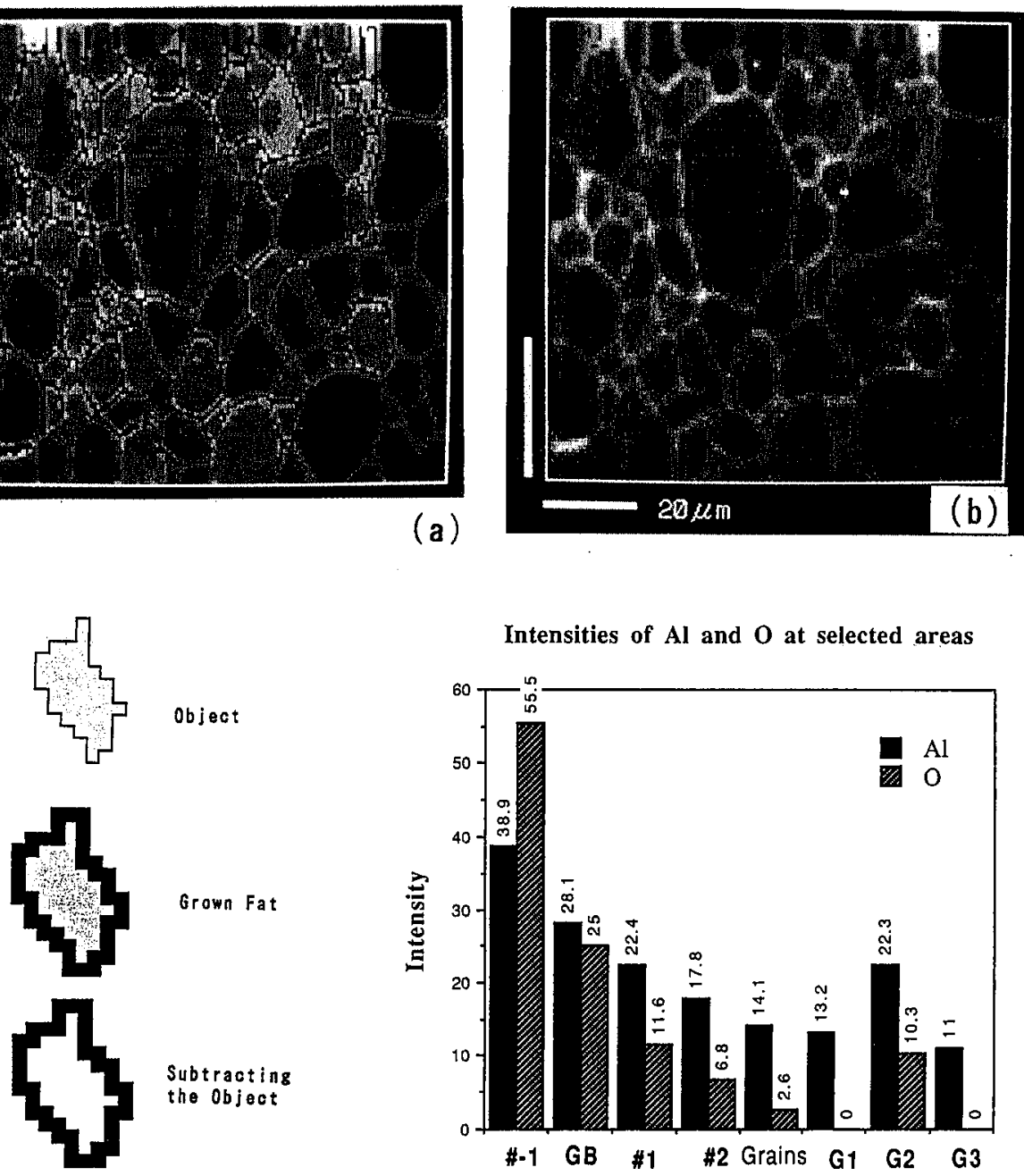

Intensities of $\mathrm{Al}$ and $\mathrm{O}$ at selected areas

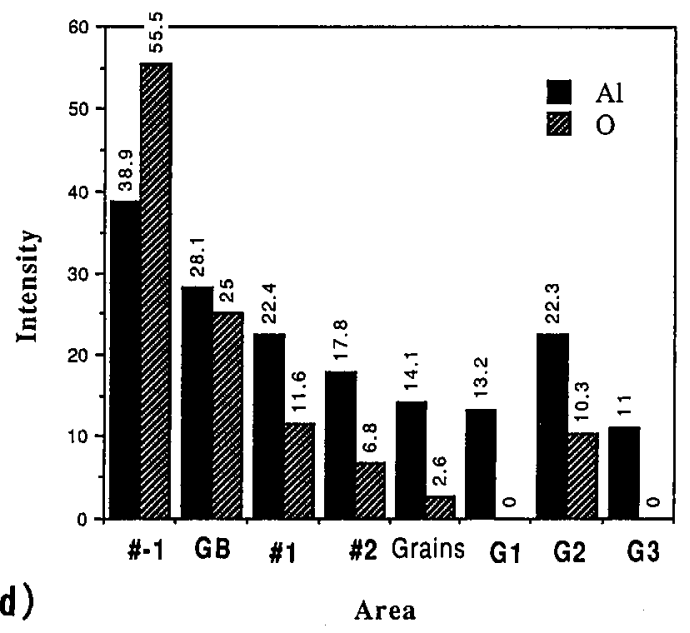

Fig. 10. Near grain boundary analysis. (a) Grain boundary image overlapped on secondary electron image. The grain boundary image is binary image extracted through processing of the secondary electron image. (b) Near-grain-boundary image overlapped on secondary electron image. (c) Procedure of extracting a near grain boundary image. (d) Auger signal intensity changes according to a distance from grain boundary. Also, intensities for grains are comparatively shown. Data for $\mathrm{Al}$ and $\mathrm{O}$. GB: intensities for all grain boundary area, \# 1: for stripe regions one pixel off from grain boundary edge, \# 2: for stripe region two pixels off from grain boundary edge, \# -1: for grain boundary area grown thin by one pixel, Grains: for all grain area, G1-G3: for grains 1-3 shown in Fig. 9a, respectively. 
in Fig. 10a. This created binary image can be used as a mask to select signals at or near the grain boundaries. That is, the intensity data for an Auger electron image is processed only when the corresponding pixel of the binary image is checked as unit. We can also make a mask comprising regions one pixel off the grain boundary edge, by further processing of the binary image. An idea is shown in Fig. 10c. The object region is expanded by one pixel outside the initial region in all directions and a striped region of one pixel width which is one pixel off from the grain boundary edge is remained by subtracting the original object from the expanded. An example is given in Fig. 10b. In the same way, we can also choose a striped region of one pixel width which is two pixel off from the grain boundary edge. Similarly we can make a striped region inside the original grain boundary by making the objects thinner. The intensities of $\mathrm{Al}$ and $\mathrm{O}$ at or near grain boundaries are compared in Fig. 10d. The bars indicate the $\mathrm{Al}$ and $\mathrm{O}$ Auger electron intensities accumulated from selected areas of the images acquired between the 4th and 14th depth levels. In this figure, the data for the grain boundary is labeled as "GB", for the stripe region one pixel off grain boundary edge as "\# 1", for the stripe region two pixels off as "\# 2", the one pixel reduced stripe area as "\# -1". The figure also includes the data for grain 1 (G1), grain 2 (G2), grain 3 (G3) and the summation of all grains (Grains). Oxygen has the highest intensity but it decays rapidly with increasing distance from the grain boundary. No $\mathrm{O}$ was detected in G1 and G3. The aluminum intensity varies similarly as the $O$ intensity, but it changes more slowly with distance than the $O$ signal. Aluminum was detected in all regions examined.

\section{Summary and discussion}

The conventional depth profiling method gives compositional information with depth on a specified spot on the specimen. When the specimen has lateral inhomogeneities, information at single spot is not enough for characterization. We have presented two methods for depth profiling that give lateral information as well. One method is to construct a cross-sectional image by repeated ion sputtering and line measurements of specified Auger signals. This method gives us depth and lateral information on element distributions. The advantage of this method is it is relatively fast. Since data is acquired in two dimensions (2-D), the data collection time except time for ion sputtering is the same as conventional Auger image acquisition. In a typical case, we will select, for example, $5000 \mathrm{~ms}(10 \mathrm{~ms}$ $\times 250$ points $\times 2$ (the " 2 " means peak and background acquisition)) as a time for data collection per line. On the other hand, in conventional depth profiling, it also requires similar time for acquisition, for example, $100 \mathrm{~ms} \times 50$ points per window. Thus, the total data collection time including the sputtering time is similar to that for conventional spot depth profiling. The second method is to collect three-dimensional (3-D) data by repeated ion sputtering and Auger image measurements (secondary electron image (SEI) can be included if necessary). While the method requires a long time for data collection, it gives us detailed information through post-processing of the 3-D data (for example, local depth profiles for a specified grain, specified region, or grain boundary). In addition, a cross-sectional image through a given line can be generated. It should be noted that the calcu- 
lated results from 3-D data by post-processing generally show good stability. Since integration of data over a specified volume in the data space enhances the signal and suppresses noise even if the signal-to-noise ratio for a single data point is poor. The above advantage is worthy of trade-off for the long data collection time in some cases. Thus, it is recommended to select an appropriate method for a given problem among the conventional, 2-D, and 3-D methods.

We have shown that image processing techniques can be utilized for selecting an area to be analyzed. These include image filtration (e.g. differentiation by Laplacian), conversion to a binary image with threshold slicing, logical processing of binary images, etc., and combinations of these methods. It has been shown that a designated grain area, grain boundary area and so on can be chosen.

We have shown two examples of application.

The 2-D method was applied to an analysis of relay contact failure. The contact electrode made of $\mathrm{Fe}-\mathrm{Ni}$ alloy coated with a $\mathrm{Au}$ thin layer was analyzed. It was known that $\mathrm{Fe}$ and $\mathrm{Ni}$ permeated the Au layer to form an oxide layer on the surface by conventional depth profiling. By cross-sectional imaging, it was shown that the permeation took place through pin holes.

The 3-D method was applied to thę analysis of a VTR head made of polycrystalline ferrite coated with a Sendust (Si-Al-Fe alloy) layer at the gap. Local area depth profiling showed that the depth profiles were not the same for each grain. Cross-sectional Auger images indicated enhanced Al signals distributing more at grain boundaries and in certain grains of small size. With the use of image procèssing method, we produced distributions of a verage signal intensities for $\mathrm{Al}$ and $O$ in grain boundary areas and in specified near-grain-boundary areas from 3-D data for the Sendust layer. Oxygen shows maximum intensity at grain boundaries and decays rapidly with distance from the grain boundaries. The Al signal behaves similarly, but decays more slowly.

Finally, we believe that 2-D and 3-D Auger analysis will be a powerful tool for surface and interface analysis of inhomogeneous specimens.

\section{References}

[1] C.A. Evans, Jr., Anal. Chem. 13, 67A (1972).

[2] S.R. Bryan, W.S. Woodward, R.W. Linton, J. Vac. Sci. Technol. A 3(6), 2102 (1985).

[3] T. Sekine, Y. Ando, Y. Sakai, Proc. JSPS 141 Committee, May 20-21, 1988, Naruto (Japan) (in Japanese), p. 13.

[4] G. Gillen, D.L. Kaiser, J.S. Wallace, Surf. Interface Anal. 14, 7 (1991).

[5] R. Van den Berghe, R. Vlaeminck, R. Van Paemel, M. Saille, Surf. Interface Anal. 8, 205 (1986).

[6] M. Murko-Jezovsek, F. Brecelj, Vacuum 37, 165 (1987); Y. Sakai, T. Sato, Y. Ando, A.D. Bounaquisti, in: Analytical Electron Microscopy, Ed. D.C. Joy, San Francisco Press, San Francisco 1987, p. 335

[7] K. Kajiwara, M. Hayakawa, Y. Kunito, Y. Ikeda, K. Hayashi, K. Aso, T. Ishida, IEEE Transactions on Magnetics 24, 6, 2620 (1988).

[8] A. Zalar, Surf. Interface Anal. 9,41 (1986). 